Pacific Journal of Mathematics

THE BOREL SPACE OF YON NEWMAN ALGEBRAS ON A 


\title{
THE BOREL SPACE OF VON NEUMANN ALGEBRAS ON A SEPARABLE HILBERT SPACE
}

\author{
EDWARD G. EFFROS
}

\begin{abstract}
Let $(S, \mathscr{S}$ ) be a Borel space (see G.W. Mackey, Borel structures in groups and their duals, Trans. Amer. Math. Soc. 85, (1957) 134-165), $\mathscr{H}$ a separable Hilbert space, $\&$ the bounded linear operators on $\mathscr{C}$ with the Borel structure generated by the weak topology, and $\mathscr{A}$ the collection of von Neumann algebras on $\mathscr{H}$. A field of $\mathscr{H}$ von Neumann algebras on $S$ is a map $s \rightarrow \mathfrak{A}(s)$ of $S$ into $\mathscr{A}$. We prove that there is a unique standard Borel structures on $\mathscr{A}$ with the property that $s \rightarrow \mathfrak{A}(s)$ is Borel if and only if there exist countably many Borel functions $s \rightarrow A_{i}(s)$ of $\mathbf{S}$ into $\&$ such that for each $s$, the operators $\mathbf{A}_{i}(s)$ generate $\mathfrak{A}(s)$. This is a consequence of the more general result that when it is provided with a suitable Borel structure, the space of weakly* closed subspaces of the dual of a separable Banach space has sufficiently many Borel choice functions.

We show that the commutant, join, and intersection operations on $\mathscr{A}$ are Borel. It follows that the Borel space of factors is standard. The relevance of $\mathscr{A}$ to the theory of group representations is also investigated.
\end{abstract}

Essentially following von Neumann [9], we say that a field $s \rightarrow \mathfrak{U}(s)$ is Borel if there exist countably many Borel functions $s \rightarrow A_{i}(s)$ of $S$ into \& such that for each $s$ the operators $A_{i}(s)$ generate $\mathfrak{U}(s)$. This definition may be regarded as somewhat artificial. Rather than state which maps of $S$ into $\mathscr{A}$ are Borel, one would conjecture that there is a standard Borel structure on $\mathscr{A}$ for which this characterization of the Borel maps of $S$ into $\mathscr{A}$ is then valid. In $\S 2$ and $\S 3$ we shall show that this is the case. The demonstration depends on two results: a theorem in [4] showing that a certain Borel structure on the closed subsets of a polonais space is standard, and Theorem 2 of this paper. In the latter we prove the existence of Borel choice functions for the weakly* closed subspaces of the dual of a separable Banach space.

The Borel space $\mathscr{A}$ is of importance in representation theory. If $G$ is a second countable locally compact group, and $G^{c}(\mathscr{L})$ are the weakly continuous unitary representions of $G$ on $\mathscr{H}$ with the weak Borel structure (see [8]), the map $L \rightarrow L(G)^{\prime}$ (prime indicates commutant) of $G^{c}(\mathscr{H})$ into $\mathscr{A}$ is Borel. By proving in $\S 3$ that the factors $\mathscr{F}$ are

Received September 21, 1964. This work has been partially supported by the National Science Foundation under grant NSF GP 1904. 
a Borel subset of $\mathscr{A}$, we obtain new proof in $\S 4$ of Dixmier's result that the factor representations $G^{f}(\mathscr{C})$ form a Borel subset of $G^{c}(\mathscr{C})$. We are also able to show that the quasi-equivalence relation is a Borel subset of $G^{c}(\mathscr{C}) \times G^{c}(\mathscr{C})$.

It is interesting to speculate about the isomorphism relation on $\mathscr{F}$. Conceivably, one might find an argument similar to those in [3] to prove that the quotient space was not smooth, and thus in particular, that there are uncountably many essentially distinct factors on $\mathscr{C}$.

We remark that an analogous problem of a "nonintrinsic" definition of structure, solved for $\mathscr{A}$ below, exists in Spanier's definition of a quasi-topology [12]. As is shown in [12], one must look for structures more general than topologies.

We are indebted to E. Alfsen and E. St $\phi$ rmer, who enabled us to simplify the proofs of Theorem 2 (by the convexity argument for the continuity of $L$ ) and Theorem 5, respectively.

2. Separable Banach spaces. Let $\mathfrak{X}$ be a separable real or complex Banach space, $\mathfrak{X}^{*}$ the dual of $\mathfrak{X}, \mathscr{N}(\mathfrak{X})$ the norm closed subspaces of $\mathfrak{X}$, and $\mathscr{W}\left(\mathfrak{X}^{*}\right)$ the weakly* closed subspaces of $\mathfrak{X}^{*}$. We wish to define a Borel structure on $\mathscr{W}\left(\mathfrak{X}^{*}\right)$. As $\mathfrak{Y} \rightarrow \mathfrak{Y} \perp$ (the annihilator of $\mathfrak{Y})$ is a one-to-one correspondence between $\mathscr{N}(\mathfrak{X})$ and $\mathscr{V}\left(\mathfrak{X}^{*}\right)$, it suffices to find a Borel structure on $\mathscr{N}(\mathfrak{X})$ and then to transfer it to $\mathscr{\mathscr { V }}\left(\mathfrak{X}^{*}\right)$.

$\mathscr{N}(x)$ is a subset of $\mathscr{C}_{0}(\mathfrak{X})$, the collection of nonempty closed subsets of the polonais space $\mathfrak{X}$. In [4] we showed that convergence of subsets in $\mathscr{C}_{0}(\mathfrak{X})$ defines a standard Borel structure on $\mathscr{C}_{0}(\mathfrak{X})$. Recalling the procedure, if $F_{\alpha}$ is a net in $\mathscr{C}_{0}(\mathfrak{X}) \operatorname{let} \lim F_{\alpha}$ be those $x$ in $x$ for which there is a net $x_{\alpha} \in F_{\alpha}$ with $x_{\alpha} \rightarrow x$. Let $\overline{\lim } F_{\alpha}$ be those $x$ in $\mathfrak{X}$ for which there is a subnet $F_{\alpha_{\beta}}$ and $x_{\alpha_{\beta}} \in F_{\alpha_{\beta}}$ with $x_{\alpha_{\beta}} \rightarrow x$. If $F \in \mathscr{C}_{0}(ख)$, we say that $F_{\alpha}$ converges to the limit $F, F_{\alpha} \rightarrow F$, if $F=\underline{\lim } F_{\alpha}=\varlimsup_{\lim } F_{\alpha}$. If $\Sigma \subseteq \mathscr{C}_{0}(\mathfrak{X})$, we let $\bar{\Sigma}$ be the limits of nets in $\Sigma$, and we say that $\Sigma$ is convergence closed if $\bar{\Sigma}=\Sigma$. The convergence closed sets form a topology, and generate a standard Borel structure on $\mathscr{C}_{0}(\mathfrak{x})$. We let $\mathscr{N}(\mathfrak{X})$ have the relative Borel structure. It is easily verified that $\mathscr{N}(\mathfrak{X})$ is convergence closed in $\mathscr{C}_{0}(\mathfrak{X})$, hence $\mathscr{N}(\mathfrak{X})$ and $\mathscr{Y}\left(X^{*}\right)$ have standard Borel structures.

If $d$ is any metric on $\mathfrak{X}$ compatible with the topology of $\mathfrak{X}, x \in \mathfrak{X}$, and $F \in \mathscr{C}_{0}(\mathfrak{x})$, define $d(x, F)=\operatorname{glb}\{d(x, y): y \in F\}$. For any positive $c$,

$$
\left\{F \in \mathscr{C}_{0}(\mathfrak{X}): d(x, F) \geqq c\right\}
$$

is convergence closed. It follows that $F \rightarrow d(x, F)$ is a Rorel function on $\mathscr{C}_{0}(\mathfrak{X})$. As in the proof of the first theorem in [4], sets of the form (1) separate points in $\mathscr{C}_{0}(\mathfrak{X})$, and thus as $\mathscr{C}_{0}(\mathfrak{X})$ is standard, generate the Borel structure. It follows that the Borel structure on 
$\mathscr{C}_{0}(\mathfrak{X})$ is the weakest for which the functions $F \rightarrow d(x, F)$ are Borel (actually it would suffice to restrict to the $x$ in a countable dense subset).

Let $d$ be the norm metric on $\mathfrak{X}$. Then for $\mathfrak{Y} \in \mathscr{W}\left(\mathfrak{X}^{*}\right), d\left(x, \mathfrak{Y}^{\perp}\right)=$ $\left\|x+\mathfrak{Y}^{\perp}\right\|$, the latter being the quotient norm in $\mathfrak{X} / \mathfrak{Y}^{\perp}$. As $\mathfrak{Y}$ is weakly* closed, $\mathfrak{Y} \perp \quad \mathfrak{Y}$, and we have a natural isometry $\left.(\mathfrak{X} / \mathfrak{Y})^{\perp}\right)^{*} \cong \mathfrak{Y}$. The corresponding isometry of $\mathfrak{X} / \mathfrak{Y}^{\perp}$ into $\mathfrak{Y} *$ is defined by $\left.x+\mathfrak{Y}\right)^{\perp} \rightarrow x \mid \mathfrak{Y}$, where $x \mid \mathfrak{Y}$ in the restriction of $x$, regarded as an element of $\mathfrak{X}^{* *}$, to Y. We conclude:

Theorem 1. Let $\mathfrak{X}$ be a separable Banach space, $\mathscr{W}\left(\mathfrak{X}^{*}\right)$ the weakly* closed subspaces of $\mathfrak{X}^{*}$. The Borel structure on $\mathscr{Y}^{-}\left(\mathfrak{X}^{*}\right)$ is standard, and may be described as the smallest structure for which the functions

$$
\mathfrak{V} \rightarrow\left\|x+\mathfrak{Y}^{\perp}\right\|=\|x \mid \mathfrak{Y}\|,
$$

$x \in \mathfrak{X}$

are Borel.

If $\mathfrak{X}$ is a real or complex separable Banach space, the weak* Borel structure on $\mathfrak{X}^{*}$ is that generated by the weak* topology. In other words, it is the smallest structure for which the functions $f \rightarrow f(x)$, $x \in \mathfrak{X}$ are Borel. Although we shall not use this fact, we remark that this structure is standard (see the proof of [8, Th. 8.1]).

Theorem 2 may be regarded as an elaborate form of the Hahn-Banach Theorem. Recalling the usual argument, suppose that $\mathfrak{X}$ is a real Banach space, and that we wish to construct a function in the closed unit ball $\mathfrak{X}_{1}^{*}$ of $\mathfrak{X}^{*}$. Suppose that $f$ has been defined on a linear subspace $\mathfrak{B}$ of $\mathfrak{X}$, and is in $\mathfrak{B}_{1}^{*}$. If we extend $f$ to the space generated by $\mathfrak{B}$ and a vector $x$, we must insist that

$$
|f(x+w)| \leqq\|x+w\|
$$

for all $w \in \mathfrak{B}$, i.e.,

$$
-\|x+u\|-f(u) \leqq f(x) \leqq\|x+v\|-f(v)
$$

for all $u, v \in \mathfrak{B}$. Let

$$
\begin{aligned}
& L(f)=\operatorname{lub}\{-\|x+u\|-f(u): u \in \mathfrak{B}\}, \\
& M(f)=\operatorname{glb}\{\|x+v\|-f(v): v \in \mathfrak{B}\} .
\end{aligned}
$$

These exist as for any $u, v \in \mathfrak{B}$,

$$
f(v-u) \leqq\|v-u\| \leqq\|x+v\|+\|x+u\|,
$$

(4) i.e.,

$$
-\|x+u\|-f(u) \leqq\|x+v\|-f(v) .
$$

Thus we may rewrite (2): 


$$
L(f) \leqq f(x) \leqq M(f) .
$$

We shall assume below that $\mathfrak{B}$ is finite dimensional, and let $\mathfrak{B}^{*}$ have the norm topology. The functions $f \rightarrow L(f)$ and $f \rightarrow M(f)$ are defined on the closed unit ball $\mathfrak{B}_{1}^{*}$. As it is the least upper bound of convex functions, $f \rightarrow L(f)$ is convex, and thus continuous on the interior of of $\mathfrak{B}_{1}^{*}$ (see $[1$, p. 92]). From

$$
M(f)=-L(-f),
$$

$f \rightarrow M(f)$ is also continuous on the interior of $\mathfrak{B}_{1}^{*}$.

TheOREM 2. Let $\mathfrak{X}$ be a separable Banach space, $\mathscr{Y}^{-}\left(\mathfrak{X}^{*}\right)$ the weakly* closed subspaces of $\mathfrak{x}^{*}$. There exist countably many Borel choice functions $f_{n}: \mathscr{W}\left(\mathfrak{X}^{*}\right) \rightarrow \mathfrak{X}^{*}$ such that for each $\mathfrak{Y} \in \mathscr{W}^{\prime}\left(\mathfrak{X}^{*}\right)$, the vectors $f_{n}(\mathfrak{Y})$ are weakly* dense in the closed unit ball $\mathfrak{V}_{1}$ of $\mathfrak{Y}$.

Proof. Suppose that $\mathfrak{X}$ is real. If $\mathfrak{Y} \in \mathscr{W}(\mathfrak{X})$, we may identify $\mathfrak{Y}$ with $\left.(\mathfrak{X} / \mathfrak{Y})^{\perp}\right)^{*}$, the norms and the weak* topologies will coincide.

For each sequence of real numbers $t=\left(t_{1}, t_{2}, \cdots\right)$ with $0 \leqq t_{i} \leqq 1$, we shall construct a function $f_{t}^{\mathfrak{Y}} \in\left(\mathfrak{X} / \mathfrak{Y}^{\perp}\right)_{i}^{*}$. Let $x_{1}, x_{2}, \cdots$ be norm dense in $\mathfrak{X}$, with $x_{1}=0$. Let $x_{n}(\mathfrak{Y})=x_{n}+\mathfrak{Y}^{\perp}$, and $\mathfrak{B}_{n}(\mathfrak{Y})$ be the linear space spanned by $x_{1}(\mathfrak{Y}), \cdots, x_{n}(\mathfrak{Y})$ in $\left.\mathfrak{X} / \mathfrak{Y}\right)^{\perp}$. Define $f_{t_{1}}^{\mathfrak{Y}}(0)=0$. Suppose that we have defined $f_{t_{1}, \cdots, t_{n}}^{\mathfrak{Y}}$ to be an element of $\mathfrak{B}_{n}(\mathfrak{Y})_{1}^{*}$. Letting $\mathfrak{B}_{n}(\mathfrak{Y})=\mathfrak{B}, f_{t_{1}, \cdots, t_{n}}^{\mathfrak{Y}}=f$, and $x_{n+1}(\mathfrak{Y})=x$ in our previous discussion, define

$$
f_{t_{1}, \cdots, t_{n+1}}^{\mathfrak{V})}(x)=t_{n+1} L(f)+\left(1-t_{n+1}\right) M(f) .
$$

If $x \in \mathfrak{B}$, letting $u=v=-x$, we have from (3), (5), and (7)

$$
-f(u) \leqq L(f) \leqq f_{t_{1}, \cdots, t_{n+1}}^{\mathfrak{Y}}(x) \leqq M(f) \leqq-f(v),
$$

i.e.,

$$
f_{t_{1}, \cdots, t_{n+1}}^{(⿰)}(x)=f(x) \text {. }
$$

Thus defining $f_{t_{1}, \ldots, t_{n+1}}^{\mathfrak{Y}}$ on $\mathfrak{V}_{n+1}(\mathfrak{Y})$ by

$$
f_{t_{1}, \cdots, t_{n+1}}^{\mathfrak{Y}}(c x+w)=c f_{t_{1}, \cdots, t_{n+1}}^{\mathfrak{Y}}(x)+f(w),
$$

we obtain an extension of $f_{t_{1}, \ldots, t_{n}}^{\mathfrak{Y}}$ to an element of $\mathfrak{B}_{n+1}(\mathfrak{Y})^{*}$. As $f=$ $f_{t_{1}, \cdots, t_{n+1}}^{\mathfrak{Z})}$ satisfies (5), it readily follows that $f_{t_{1}, \ldots, t_{n+1}}^{\mathfrak{Y}}$ is in $\mathfrak{B}_{n+1}(\mathfrak{Y})_{1}^{*}$. Define $f_{t}^{\mathfrak{Y}}$ on the space spanned by the $x_{n}(\mathfrak{Y})$ to be the union of the functions $f_{t_{1}, \cdots, t_{n}}^{\text {y }}$. This extends by continuity to an element of $\left(\mathfrak{X} / \mathfrak{Y}^{\perp}\right)_{1}^{*}$.

It is clear that any function in $\left.(\mathfrak{X} / \mathfrak{Y})^{\perp}\right)_{1}^{*}$ must have the form $f_{t}^{\mathfrak{Y}}$ 
for some sequence $t=\left(t_{1}, t_{2}, \cdots\right)$. We claim that the countable family of functions $f_{r}^{\mathfrak{V}}, r=\left(r_{1}, r_{2}, \cdots\right)$ with the $r_{i}$ rational, and all but a finite number equal to 0 , are weakly* dense in $\left(\mathfrak{X} / \mathfrak{Y}^{\perp}\right)_{1}^{*}$. It suffices to prove that for all $n$, the functions $f_{r_{1}, \ldots, r_{n}}^{\mathfrak{Y}}$ are weakly*, or equivalently, norm dense in the interior of $\left(\mathfrak{B}_{n}(\mathfrak{Y})\right)_{1}^{*}$. This is trivial if $n=1$. Suppose that it is true for $n$. If $g \in \mathfrak{B}_{n+1}(\mathfrak{Y})^{*}$ and $\|g\| \leqq 1$, let $f$ be the restriction of $g$ to $\mathfrak{B}_{n}(\mathfrak{Y})$. From our hypothesis and the earlier discussion, we may select rationals $r_{1}, \cdots, r_{n}$ with $f_{r_{1}, \ldots, r_{n}}^{\mathfrak{Y}}$ close to $f$ in the norm topology, and $L\left(f_{r_{1}, \ldots, r_{n}}^{\mathfrak{Y}}\right)$ and $M\left(f_{r_{1}, \ldots, r_{n}}^{\mathfrak{Y}}\right)$ close to $L(f)$ and $M(f)$, respectively. Thus by a suitable choice of $r_{n+1}$, we obtain

$$
f_{r_{1}, \ldots, r_{n+1}}^{\mathfrak{Y}}\left(x_{n+1}(\mathfrak{Y})\right)
$$

close to $g\left(x_{n+1}(\mathfrak{Y})\right)$.

For any sequence $\left(t_{1}, t_{2}, \cdots\right)$ we have that $\mathfrak{Y} \rightarrow f_{t}^{\mathfrak{Y})}\left(x_{n}\right)$ is Borel (regarding $f_{t}^{\mathfrak{V}}$ as an element of $\mathfrak{Y}$ ). This is trivial if $n=1$. Suppose that it is true for $k \leqq n$. Then

$$
\begin{aligned}
f_{t}^{\mathfrak{Y}}\left(x_{n+1}\right) & =f_{t_{1}, \cdots, t_{n+1}}^{\mathfrak{Y}}\left(x_{n+1}(\mathfrak{Y})\right) \\
& =t_{n+1} L\left(f_{t_{1}, \ldots, t}^{\mathfrak{Y}, \ldots, t}\right)+\left(1-t_{n+1}\right) M\left(f_{t}^{\mathfrak{Y}}, \ldots, t\right)
\end{aligned}
$$

If $\mathfrak{B}_{n}$ is the linear span of $x_{1}, \cdots, x_{n}$,

$$
L\left(f_{t_{1}, \ldots, t_{n}}^{\mathfrak{Y}}\right)=\operatorname{lub}\left\{-\left\|x_{n+1}+u+\mathfrak{Y}^{\perp}\right\|-f_{t}^{\mathfrak{Y}}(u): u \in \mathfrak{B}_{n}\right\} .
$$

From Theorem 1 and the induction hypothesis,

$$
\mathfrak{Y} \rightarrow-\left\|x_{n+1}+u+\mathfrak{Y}^{\perp}\right\|-f_{t}^{\mathfrak{Y}}(u)
$$

is Borel for any $u \in \mathfrak{B}_{n}$. Restricting to $u$ that are rational linear combinations of the $x_{k}$ for $k \leqq n, \mathfrak{Y} \rightarrow L\left(f_{t_{1}, \ldots, t_{n}}^{\mathfrak{Y}}\right)$ is the least upper bound of a countable number of Borel functions, and is thus Borel. From (6) and (8), $\mathfrak{Y} \rightarrow f_{t}^{\mathfrak{Y}}\left(x_{n+1}\right)$ is Borel. For any $x \in \mathfrak{X}, \mathfrak{Y} \rightarrow f_{t}^{\mathfrak{Y}}(x)$ is a limit of functions of the form $\mathfrak{Y} \rightarrow f_{t}^{\mathfrak{Y}}\left(x_{n}\right)$, and hence is Borel. Thus $\mathfrak{Y} \rightarrow f_{t}^{\mathfrak{Y}}$ is Borel.

Finally, suppose that $\mathfrak{X}$ is a complex Banach space. Letting $\mathfrak{X}_{R}$ be the corresponding real Banach space, $\mathscr{N}(\mathfrak{X})$ is a convergence closed subset of $\mathscr{N}\left(\mathfrak{X}_{R}\right)$. Define a map of $\mathscr{W}\left(\mathfrak{X}^{*}\right)$ into $\mathscr{W}\left(\left(\mathfrak{X}_{R}\right)^{*}\right)$ by $\mathfrak{Y} \rightarrow \operatorname{Re} \mathfrak{Y}$, where the latter consists of all real functions $\operatorname{Re} f$ with $f \in \mathfrak{Y}$ ) (the customary argument shows that $f \rightarrow \operatorname{Re} f$ is an isometry of $\mathfrak{X}^{*}$ onto $\left.\left(\mathfrak{X}_{R}\right)^{*}\right)$. For $3 \in \mathscr{N}(\mathfrak{X}), \operatorname{Re}\left(3^{\perp}\right)=3^{\perp}$, where annihilators are taken in $\mathfrak{X}^{*}$ and $\left(\mathfrak{X}_{R}\right)^{*}$, respectively. It follows that $\mathfrak{Y} \rightarrow \operatorname{Re} \mathfrak{Y}$ defines a Borel isomorphism of $\mathscr{W}\left(\mathfrak{X}^{*}\right)$ onto a Borel subset of $\mathscr{W}\left(\left(\mathfrak{X}_{R}\right)^{*}\right)$. Choose real choice functions $f_{n}$ : $\mathscr{W}\left(\left(\mathfrak{X}_{R}\right)^{*}\right) \rightarrow\left(\mathfrak{X}_{R}\right)^{*}$ with $f_{n}(\mathfrak{Y})$ weakly* dense in $\mathfrak{Y}_{1}$ for each $\mathfrak{Y} \in \mathscr{W}\left(\left(\mathfrak{X}_{R}\right)^{*}\right)$. Let $g_{n}$ : $\mathscr{W}\left(\mathfrak{X}^{*}\right) \rightarrow \mathfrak{X}^{*}$ be the corresponding complex functions, i.e., for $\mathfrak{Y} \in \mathscr{W}\left(\mathfrak{X}^{*}\right)$ and $x \in \mathfrak{X}$, let 


$$
g_{n}(\mathfrak{Y})(x)=f_{n}(\operatorname{Re} \mathfrak{Y})(x)-i f_{n}(\operatorname{Re} \mathfrak{Y})(i x) \text {. }
$$

Then $\operatorname{Re} g_{n}(\mathfrak{Y})=f_{n}(\operatorname{Re} \mathfrak{Y}) \in(\operatorname{Re} \mathfrak{Y})_{1}$, implies $g_{n}(\mathfrak{Y}) \in \mathfrak{Y}_{1}$. Given an arbitrary $g \in \mathfrak{Y}_{1}, x_{1}, \cdots, x_{k} \in \mathfrak{X}$, and $\varepsilon>0$, choose an $f_{n}$ with

$$
\begin{gathered}
\left|f_{n}(\operatorname{Re} \mathfrak{Y})\left(x_{j}\right)-\operatorname{Re} g\left(x_{j}\right)\right|<\varepsilon \\
\left|f_{n}(\operatorname{Re} \mathfrak{Y})\left(i x_{j}\right)-\operatorname{Re} g\left(i x_{j}\right)\right|<\varepsilon,
\end{gathered}
$$

for $j=1, \cdots, k$. Then as

$$
g(x)=\operatorname{Re} g(x)-i \operatorname{Re} g(i x),
$$

we have

$$
\left|g_{n}(\mathfrak{Y})\left(x_{\jmath}\right)-g\left(x_{j}\right)\right|<2 \varepsilon
$$

for $j=1, \cdots, k$. Thus the $g_{n}(\mathfrak{Y})$ are weakly* dense in $\mathfrak{Y}_{1}$. Clearly the $g_{n}$ are Borel.

Corollary. If $(S, \mathscr{S})$ is a Borel space, then a map $s \rightarrow \mathfrak{Y}(s)$ of $S$ into $\mathscr{W}\left(\mathfrak{X}^{*}\right)$ is Borel if and only if there exist countably many Borel functions $s \rightarrow f_{n}^{s}$ of $S$ into $\mathfrak{X}^{*}$, such that for each $s$, the vectors $f_{n}^{s}$ are weakly dense in $\mathfrak{Y}(s)_{1}$.

Proof. If $s \rightarrow \mathfrak{Y}(s)$ is Borel, the functions $f_{n}^{s}$ are obtained by composing this map with the choice functions of Theorem 2. Conversely, if such functions exist, we have from the isometry

$$
\begin{gathered}
\mathfrak{Y}(s) \cong\left(\mathfrak{X} / \mathfrak{Y}(s)^{\perp}\right)^{*}, \\
\left\|x+\mathfrak{Y}(s)^{\perp}\right\|=\sup \left\{\left|f_{i}^{s}(x)\right|: i=1,2, \cdots\right\}
\end{gathered}
$$

for each $x \in \mathfrak{X}$. Thus $s \rightarrow\left\|x+\mathfrak{Y}(s)^{\perp}\right\|$ is Borel for each $x \in \mathfrak{X}$, and by Theorem $1, s \rightarrow \mathfrak{Y}(s)$ is Borel.

3. Von Neumann algebras. Let $\mathscr{C}, \mathcal{L}, \mathscr{A}$, and $\mathscr{F}$ be as in $\S 1$. We have that $\mathbb{R}=\left(\mathbb{R}_{*}\right)^{*}$, where $\mathbb{R}_{*}$ is the separable Banach space of ultra-weakly continuous functions on $\mathbb{L}$ (or by a natural identification, the trace class operators with a suitable norm-see [10]). The ultra-weak and weak* topologies coincide on \&. Thus letting $\mathscr{W}(\mathbb{R})$ be the ultra-weakly closed subspaces of $\mathbb{L}$, we may give it the Borel structure described in $\S 2$.

If $\mathfrak{Y} \in \mathscr{W}(\mathfrak{Q})$, write $\mathfrak{Y} *$ and $\mathfrak{Y}^{\prime}$ for the adjoints of elements in $\mathfrak{Y}$, and the commutant of $\mathfrak{Y}$, respectively. The proof of the following theorem is largely patterned after that of [6, Th. 2.8].

THEOREM 3. $\mathfrak{Y} \rightarrow \mathfrak{Y} *$ and $\mathfrak{Y} \rightarrow \mathfrak{Y}^{\prime}$ de fine Borel transformations of 
$\mathscr{W}(\mathfrak{R})$.

Proof. For $f \in \mathbb{R}_{*}$, define $f^{*} \in \mathfrak{R}_{*}$ by $f^{*}(A)=\overline{f\left(A^{*}\right)}$, the bar indicating complex conjugate. This is an isometry of $\mathfrak{Q}_{*}$, hence the transformation $\mathfrak{B} \rightarrow \mathfrak{B}^{*}$ on $\mathscr{N}(\mathbb{R})$ is a homeomorphism (in the sense of convergence), and a Borel isomorphism. For $\left.\mathfrak{Y} \in \mathscr{Y}(\mathfrak{Q}),\left(\mathfrak{Y}^{\perp}\right)^{*}=(\mathfrak{Y})^{*}\right)^{\perp}$, i.e., the adjoint operation on $\mathscr{N}\left(\mathbb{2}^{*}\right)$ is carried into that on $\mathscr{W}^{\prime}(\mathbb{Q})$, and thus is a Borel isomorphism on the latter.

From Theorem 2, we may let $\mathfrak{Y} \rightarrow A_{n}^{\mathfrak{Y}}$ be Borel choice functions on $\mathscr{W}(\mathbb{\Omega})$ with $A_{n}^{\mathfrak{V})}$ ultra-weakly dense in $\mathfrak{Y}_{1}$. We have

$$
\mathfrak{Y}^{\prime}=\left\{B \in \mathfrak{L}: B A_{n}^{\mathfrak{Y}}-A_{n}^{\mathfrak{Y} B}=0 \text { for } n=1,2, \cdots\right\} .
$$

Let $\mathfrak{M}$ and $\mathfrak{M}_{*}$ be the sequences $\left(A_{n}\right)$ and $\left(f_{n}\right)$ of elements in $\mathfrak{Z}$ and $\mathfrak{L}_{*}$, respectively, with $\sup \left\{\left\|A_{n}\right\|: n=1,2, \cdots\right\}<\infty$ and $\sum_{n=1}^{\infty}\left\|f_{n}\right\|<\infty$. With the norms $\left\|\left(A_{n}\right)\right\|=\sup \left\{\left\|A_{n}\right\|: n=1,2, \cdots\right\}$ and $\left\|\left(f_{n}\right)\right\|=$ $\sum_{n=1}^{\infty}\left\|f_{n}\right\|$, $\mathfrak{M}$ and $\mathfrak{M}_{*}$ are Banach spaces, and defining $\left(f_{n}\right)\left(\left(A_{n}\right)\right)=$ $\sum_{n=1}^{\infty} f_{n}\left(A_{n}\right)$, $\mathfrak{M}$ may be identified with the dual of $\mathfrak{M}_{*}$. We have

$$
\mathfrak{Y}^{\prime}=\operatorname{kernel} T^{\mathfrak{y}},
$$

where $T^{\mathfrak{Y}:} \mathfrak{L} \rightarrow \mathfrak{M}$ is defined by

$$
T^{\mathfrak{Y}}(B)=\left(B A_{n}^{\mathfrak{Y}}-A_{n}^{\mathfrak{Y}} B\right) .
$$

we claim that $T^{\mathscr{V}}$ is continuous in the weak ${ }^{*}$ topologies. If $\left(f_{n}\right) \in \mathfrak{M}_{*}$,

$$
\left(f_{n}\right) T^{\mathfrak{Y}}(B)=\sum_{n=1}^{\infty} g_{n}(B),
$$

where $g_{n}(B)=f_{n}\left(B A_{n}^{\mathfrak{Y}}-A_{n}^{\mathfrak{Y}} B\right)$. The partial sums $\sum_{n=1}^{N} g_{n}$ are weakly* continuous, and converge uniformly on the unit ball $\mathbb{Q}_{1}$ of $\mathbb{R}$, as if $B \in \mathfrak{R}_{1}$,

$$
\left|\sum_{n=N+1}^{\infty} g_{n}(B)\right| \leqq 2 \sum_{n=N+1}^{\infty}\left\|f_{n}\right\| \text {. }
$$

It follows that $B \rightarrow\left(f_{n}\right) T^{\mathfrak{g}}(B)$ is continuous on $\mathfrak{Q}_{1}$, and thus on $\mathbb{2}$ (see [2, p. 41]). Define $T_{*}^{\mathfrak{Y}}: \mathfrak{M}_{*} \rightarrow \mathfrak{R}_{*}$ by

$$
T_{*}^{\mathfrak{V}}\left(\left(f_{n}\right)\right)(B)=\left(f_{n}\right)\left(T^{\mathfrak{V}}(B)\right) \text {. }
$$

We have that (kernel $\left.T^{\mathfrak{V}}\right)^{\perp}$ is the closure of the range of $T_{*}^{\mathfrak{Y}}$. Thus letting $B_{i}$ be ultra-weakly dense in $\mathfrak{Q}_{1}$ and $g_{j}=\left(f_{n}^{\jmath}\right)$ be norm dense in $\mathfrak{M}_{*}$, we have for any $f \in \mathfrak{L}_{*}$,

$$
\left\|f+\left(\mathfrak{Y}^{\prime}\right)^{\perp}\right\|=\operatorname{glb}\left\{\left\|f+T \stackrel{\mathfrak{g}}{*}\left(g_{j}\right)\right\|, j=1,2, \cdots\right\}
$$

where 


$$
\begin{aligned}
\| f & +T_{*}^{\mathfrak{Y}}\left(g_{j}\right) \|=\operatorname{lub}\left\{\left|f\left(B_{i}\right)+T_{*}^{\mathfrak{V}}\left(g_{j}\right)\left(B_{i}\right)\right|: i=1,2, \cdots\right\} \\
& =\operatorname{lub}\left\{\left|f\left(B_{i}\right)+\sum_{n=1}^{\infty} f_{n}^{j}\left(B_{i} A_{n}^{\mathfrak{⿰}}-A_{n}^{\mathfrak{श}} B_{i}\right)\right|: i=1,2, \cdots\right\} .
\end{aligned}
$$

As $\mathfrak{Y} \rightarrow A_{\mu}^{\mathfrak{Y}}$ is ultra-weakly Borel, $\mathfrak{Y} \rightarrow\left\|f+\left(\mathfrak{Y}^{\prime}\right)^{\perp}\right\|$ is Borel, and as $f$ is arbitrary, we have from Theorem 1 that $\mathfrak{Y} \rightarrow \mathfrak{Y}^{\prime}$ is Borel.

Corollary 1. A is a Borel subset of $\mathscr{W}$ (Y)), and thus is standard under tine relative Borel structure.

Proof. A consists of the $\mathfrak{Y} \in \mathscr{W}(\mathbb{Q})$ invariant under the Borel transformations $\mathfrak{Y} \rightarrow \mathfrak{Y} *$ and $\mathfrak{Y} \rightarrow \mathfrak{Y}^{\prime \prime}$. In general say that $\theta$ is a. Borel transformation of Eorel space $(S, \mathscr{S})$. If $\Delta$ is the diagonal of $S \times S$, and $\theta \times \iota: S \rightarrow S \times S$ is defined by $\theta \times \iota(s)=(\theta(s), s)$, we have

$$
\{s \in S: \theta(s)=s\}=(\theta \times c)^{-1}(d) .
$$

Thus if $(S, \mathscr{S})$ is standard, $\Delta$ is a Borel subset of $S \times S$, and the set of fixed points of $\theta$ is Borel.

Given von Neumann algebras $\mathfrak{A}$ and $\mathfrak{B}$, we let $\mathfrak{A} \vee \mathfrak{B}$ denote the von Neumann algebra generated by $\mathfrak{A}$ and $\mathfrak{B}$. Providing $\mathscr{A} \times \mathscr{A}$ with the product structure,

Corollary 2. The maps of $\mathscr{A} \times \mathscr{A}$ into $\mathscr{A}$ defined by $(\mathfrak{U}, \mathfrak{B}) \rightarrow \mathfrak{A} \cap \mathfrak{B}$ and $(\mathfrak{A}, \mathfrak{B}) \rightarrow \mathfrak{H} \vee \mathfrak{B}$ are Borel.

Proof. As $\mathfrak{A} \cap \mathfrak{B}=\left(\mathfrak{U}^{\prime} \vee \mathfrak{B}^{\prime}\right)^{\prime}$, it suffices to prove the second assertion. From Theorem 2 , there exist Borel choice functions $A_{i}: \mathscr{A} \rightarrow \mathbb{R}$ with $A_{i}(\mathfrak{A})$ ultra-weakly dense in $\mathfrak{A}_{1}$, for each $\mathfrak{A} \in \mathscr{A}$. For each pair $(\mathfrak{U}, \mathfrak{B}) \in \mathscr{A} \times \mathscr{A}$, let $\mathscr{C}(\mathfrak{U}, \mathfrak{B})$ be the self-adjoint linear algebra generated by the elements $A_{i}(\mathfrak{H})$ and $A_{j}(\mathfrak{B})$. Let $B_{k}(\mathfrak{H}, \mathfrak{B})$ be an enumeration of the finite complex rational combinations of finite products of the elements $A_{i}(\mathfrak{Q}), A_{j}(\mathfrak{B})$ and their adjoints. The $B_{k}(\mathfrak{I}, \mathfrak{B})$ are norm dense in $\mathscr{C}(\mathfrak{A}, \mathfrak{B})$, hence defining $B_{k}^{\prime}(\mathfrak{A}, \mathfrak{B})=B_{k}(\mathfrak{A}, \mathfrak{B})$ if $\left\|B_{k}(\mathfrak{A}, \mathfrak{B})\right\| \leqq 1$, and $B_{k}^{\prime}(\mathfrak{R}, \mathfrak{B})=0$ otherwise, the $B_{k}^{\prime}(\mathfrak{A}, \mathfrak{B})$ are norm dense in $\mathscr{C}(\mathfrak{U}, \mathfrak{B})_{1}$. From the Kaplansky Density Theorem, the latter is ultra-weakly dense in $(\mathfrak{A} \vee \mathfrak{B})_{1}$. As $(\mathfrak{A}, \mathfrak{B}) \rightarrow B_{k}^{\prime}(\mathfrak{A}, \mathfrak{B})$ are Borel, our assertion follows from the corollary to Theorem 2.

Corollary 3. $\mathscr{F}$ is a Borel subset of $\mathscr{A}$, and thus is standard in the relative Borel structure.

Proof. Let $\Im$ be the von Neumann algebra on $\mathscr{X}$ consisting of complex multiples of the identity operator. Then $\mathscr{F}^{-}$is the inverse 
image of the element $\mathfrak{\Im}$ under the Borel map of $\mathscr{A}$ into $\mathscr{A}$ defined by $\mathfrak{A} \rightarrow \mathfrak{A} \cap \mathfrak{U}^{\prime}$.

The argument used in the proof of Corollary 2 shows that a map $s \rightarrow \mathfrak{U}(s)$ of a Borel space $(S, \mathscr{S})$ into $\mathscr{A}$ is Borel if and only if there exist Borel functions $s \rightarrow A_{i}(s)$ of $S$ into $\mathbb{Q}$ such that the $A_{i}(s)$ generate $\mathfrak{H}(s)$. Thus we have recaptured the original definition of $\S 1$.

In direct integral theory, it is of some importance to know that various other subsets of $\mathscr{A}$ are measurable (see [9, 11]). We suspect that constructive procedures similar to that used in Theorem 2, would enable one to show that many of these sets are Borel.

4. Representation spaces. Let $\mathscr{C}, \mathfrak{R}, \mathscr{A}$, and $\mathscr{F}$ be as above, and $G$ be a second countable locally compact group (an analogous theory exists for separable $C^{*}$-algebras). Let $G^{c}(\mathscr{H})$ be the weakly continuous unitary representations of $G$ on $\mathscr{C}$, with the standard Borel structure defined by Mackey (see [8]). Let $G^{f}(\mathscr{H})$ be the subset of factor representations, i.e. those representations $L \in G^{c}(\mathscr{H})$ with $L(G)^{\prime}$ a factor von Neumann algebra.

If $L, M \in G^{c}(\mathscr{H})$, let $\Re(L, M)$ be the ring of intertwining operators for $L$ and $M$, i.e., those $B \in \mathfrak{Q}$ with $B L(t)=M(t) B$ for all $t \in G$. In particular, $\Re(L, L)=L(G)^{\prime}$. As was the case for Theorem 3 , the following is simply a refinement of [6, Th. 2.8].

THEOREM 4. The map $G^{c}(\mathscr{H}) \times G^{c}(\mathscr{H}) \rightarrow G^{c}(\mathscr{H})$ defined by $(L, M) \rightarrow \Re(L, M)$ is Borel.

Proof. Let $t_{n}$ be dense in $G$, and define $\mathfrak{M}$ and $\mathfrak{M}_{*}$ as in the proof of Theorem 3. Defining $S^{(L, M)}: \mathbb{L} \rightarrow \mathfrak{M}$ by

$$
S^{(L, M I)}(B)=\left(B L\left(t_{n}\right)-M\left(t_{n}\right) B\right),
$$

we have that

$$
\Re(L, M)=\operatorname{kernel} S^{(L, M)},
$$

and that $S^{(L, M)}$ is continuous in the weak* topologies. $S^{(L, M L)}$ is the adjoint of a map $S_{*}^{(L, M)}: \mathfrak{M}_{*} \rightarrow \mathfrak{R}_{*}$, and choosing $B_{i}$ ultra-weakly dense in $\mathfrak{R}_{1}$, and $g_{j}=\left(f_{j}^{n}\right)$ norm dense in $\mathfrak{M}_{*}$, we have for any $f \in L_{*}$,

$$
\left\|f+\Re(L, M)^{\perp}\right\|=\operatorname{glb}\left\{\left\|f+S_{*}^{(L, M)}\left(g_{j}\right)\right\|: j=1,2, \cdots\right\},
$$

where

$$
\begin{aligned}
\| f & +S_{*}^{(L, M)}\left(g_{j}\right) \|=\operatorname{lub}\left\{\mid f\left(B_{i}\right)\right. \\
& \left.+\sum_{n=1}^{\infty} f_{j}^{n}\left(B_{i} L\left(t_{n}\right)-M\left(t_{n}\right) B_{i}\right) \mid: i=1,2, \cdots\right\} .
\end{aligned}
$$


$(L, M) \rightarrow f_{j}^{n}\left(B_{i} L\left(t_{n}\right)-M\left(t_{n}\right) B_{i}\right)$ is Borel when $G^{c}(\mathscr{C}) \times G^{c}(\mathscr{C})$ is given the product of the Mackey Borel structures, as any ultra-weakly continuous function is a norm limit of weakly continuous functions. It follows that $(L, M) \rightarrow\left\|f+\Re(L, M)^{\perp}\right\|$ is Borel, and from Theorem $1,(L, M) \rightarrow \Re(L, M)$ is Borel. Borel

Corollary 1. The map $G^{c}(\mathscr{C}) \rightarrow \mathscr{A}$ defined by $L \rightarrow L(G)^{\prime}$ is

CoROLlary 2. (This was first proved by J. Dixmier-see [5, Theorem 1].) The set $G^{f}(\mathscr{H})$ of factor representation of $G$ forms a Borel subset of $G^{c}(\mathscr{H})$, and thus is standard under the relative Borel structure.

Following Mackey (see [7]), if $L, M \in G^{c}(\mathscr{C})$, we say that $L$ is covered by $M, L \prec M$, if very subrepresentation of $L$ contains a subrepresentation that is unitarily equivalent to a subrepresentation of $M$. $L$ is quasi-equivalent to $M, L \sim M$, if $L \prec M$ and $M \prec L$.

If $E$ is a projection in $L(G)^{\prime}$, and $E \neq 0$, let $L^{E}$ denote the corresponding subrepresentation of $G$ on the range of $E$. If there exists a projection $E \in L(G)^{\prime}$ with $E \neq 0$ and $L^{E} \prec M$, let $C(L, M)$ be the least upper bound of all such projections. Otherwise, let $C(L, M)=0$. $C(L, M)$ is an element of $L(G)^{\prime} \cap L(G)^{\prime \prime}$.

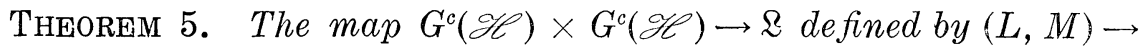
$C(L, M)$ is Borel.

Proof. If $A \in \mathbb{R}$, let $E_{A}$ and $F_{A}$ be the projections on the closure of the range, and the orthogonal complement of the kernel of $A$. If $A \in \Re(L, M)$, then $F_{A} \in L(G)^{\prime}$ and $E_{A} \in M(G)^{\prime}$. If $A \neq 0$, and $U$ is the partial isometry in the polar decomposition of $A$ with $U^{*} U=F_{A}$, then $U$ determines a unitary equivalence of $L^{F_{A}}$ and $M^{E_{A}}$, and $F_{A} \leqq C(L, M)$. From Theorems 4 and 2, there exist Borel functions $A_{i}(L, M)$ that are ultra-weakly dense in the unit ball of $\Re(L, M)$ for each $L$ and $M$. We claim that

$$
C(L, M)=\bigvee_{i=1}^{\infty} F_{A_{i}(L, M)},
$$

where on the right we have taken the least upper bound in the complete projection lattice of $L(G)^{\prime}$.

Suppose that there exist $L$ and $M$ with

$$
F=C(L, M)-\bigvee_{i=1}^{\infty} F_{A_{i}(L, M)} \neq 0 .
$$


As $\quad L^{F} \prec M$, there exists a projection $F_{0} \leqq F$ with $F_{0} \neq 0$ and $F_{0}=U^{*} U$ where $U \in \Re(L, M)$. Choosing $i_{k}$ for which $A_{i_{k}}(L, M) \rightarrow U$ ultra-weakly,

$$
0=A_{i_{k}}(L, M) F_{0} \rightarrow U F_{0}=F_{0}
$$

a contradiction.

The map of $\mathbb{Q}$ into itself defined by $A \rightarrow F_{A}$ is Borel. To see this, note that $A \rightarrow A^{*} A$ is weakly Borel, as if $x, y \in \mathscr{H}$, letting $x_{i}$ be an orthonormal basis we have

$$
A^{*} A x \cdot y=\sum_{i=1}^{\infty}\left(A x \cdot x_{i}\right)\left(A y \cdot x_{i}\right)^{-} .
$$

A similar expansion shows that for positive integers $n, A \rightarrow A^{n}$ is Borel, hence for any polynominal $p, A \rightarrow p(A)$ is Borel. Suppose that $f$ is a bounded real Borel function on the reals, and that there is a sequence of real polynomials $p_{n}$ converging to $f$ point-wise, uniformly bounded on compact sets. If $A$ is a self-adjoint element in $\mathbb{R}$, we have from spectral theory that $p_{n}(A) \rightarrow f(A)$ weakly. Thus $A \rightarrow f(A)$ is Borel. Letting $g$ be the characteristic function of the open set $(0, \infty)$, $A \rightarrow F_{A}=g\left(\left(A^{*} A\right)^{1 / 2}\right)$ is Borel.

For all $i,(L, M) \rightarrow F_{A_{i}(L, M)}$ is Borel. If $F_{1}, \cdots, F_{n}$ are propections, then

hence

$$
F_{1} \vee \cdots \vee F_{n}=F_{\left(F_{1}+\cdots+F_{n}\right)},
$$

$$
(L, M) \rightarrow \bigvee_{i=1}^{n} F_{A_{i}(L, M)}
$$

is Borel. As the projections $\bigvee_{i=1}^{n} F_{A_{i}(L, M)}$ converge weakly to $\bigvee_{i=1}^{\infty} F_{A_{i}(L, M)}$, we conclude from (9) that $(L, M) \rightarrow C(L, M)$ is Borel.

Ernest remarked in the proof of [5, Prop. 2] that the quasiequivalence relation on $G^{f}(\mathscr{H})$ is a Borel subset of $G^{f}(\mathscr{C}) \times G^{f}(\mathscr{H})$. The above theorem implies:

COROLlaRY 1. The covering and quasi-equivalence relations are Borel subsets of $G^{c}(\mathscr{H}) \times G^{c}(\mathscr{H})$.

COROLlary 2. The quasi-equivalence class $[L]$ of a representation $L$ in $G^{c}(\mathscr{H})$ is a Borel subset of $G^{c}(\mathscr{H})$.

Proof. Let $\pi_{i}: G^{c}(\mathscr{H}) \times G^{c}(\mathscr{H}) \rightarrow G^{c}(\mathscr{H}), i=1,2$, be the projections on the first and second co-ordinates. Then $[L]=\pi_{2}\left(\pi_{1}^{-1}(L) \cap \sim\right)$, and as $\pi_{2}$ is one-to-one on $\pi_{1}^{-1}(L) \cap \sim$, and the latter is standard, $[L]$ is Borel.

It would seem likely that the unitary equivalence relation is also a Borel subset of $G^{c}(\mathscr{H}) \times G^{c}(\mathscr{H})$. Presumably one must prove the 
existence of a Borel choice function on spaces of the form $\Re(L, M)$, that selects a unitary operator when such exists. If unitary equivalence were a Borel set, it would follow that the representations $L \in G^{c}(\mathscr{C})$ with $L(G)^{\prime}$ finite was also Borel. It should be noted that the unitary analogue of Corollary 2 above is true (see [3, Lemma 2.4]). If $G$ is the free group on countably many generators, the map described in Corollary 1 of Theorem 4 is onto. As the given structure and the corresponding quotient structure on $\mathscr{A}$ must coincide, a subset of $\mathscr{A}$ will be Borel if and only if the inverse image in $G^{c}(\mathscr{H})$ is Borel.

\section{BIBLIOGRAPHY}

1. N. Bourbaki, Espaces vectoriels topologiques, Act. Sci. Ind., no. 1189, Hermann, (1953).

2. J. Dixmier, Les algèbres d'opérateurs dans l'espace hilhertien, Gauthier-Villars, Paris, 1957.

3. E. Effros, Transformation groups and $C^{*}$-algebras, to appear.

4. - Convergence of closed subsets in a topological space, to appear.

5. J. A. Ernest, A decomposition theorem for unitary representations of locally compact groups, Trans. Amer. Math. Soc. 104 (1962), 252-277.

6. G. W. Mackey, Induced representations of locally compact groups II, Ann. of Math. 58 (1953), 193-221.

7. - The theory of group representations (notes by Fell and Lowdenslager), Univ. of Chicago, Lecture Notes, 1955.

8. - Borel structures in groups and their duals, Trans. Amer. Math. Soc. 85 (1957), 134-165.

9. J. von Neumann, On rings of operators. Reduction theory. Ann. of Math. 50 (1949), 401-485.

10. R. Schatten, A theory of cross spaces, Ann. of Math. Studies, no. 26, Princeton, 1959.

11. J. Schwartz, Type II factors in a central decomposition, Comm. Pure Appl. Math. 16 (1963), 247-252.

12. E. Spanier, Quasi-topologies, Duke Math. J. 30 (1963), 1-14.

COLUMBia UNIVERSITY

UNIVERSITY OF OSLO

University of PENNSYlVANIA 


\title{
PACIFIC JOURNAL OF MATHEMATICS
}

\author{
EDITORS
}

\author{
H. SAmelson \\ Stanford University \\ Stanford, California \\ R. M. Blumenthal \\ University of Washington \\ Seattle, Washington 98105
}

\author{
J. DugundjI \\ University of Southern California \\ Los Angeles, California 90007
}

*Richard Arens

University of California

Los Angeles, California 90024

\section{ASSOCIATE EDITORS}
E. F. BECKENBACH
B. H. NeUmanN
F. WOLF
K. YoSIDA

\section{SUPPORTING INSTITUTIONS}

\author{
UNIVERSITY OF BRITISH COLUMBIA \\ CALIFORNIA INSTITUTE OF TECHNOLOGY \\ UNIVERSITY OF CALIFORNIA \\ MONTANA STATE UNIVERSITY \\ UNIVERSITY OF NEVADA \\ NEW MEXICO STATE UNIVERSITY \\ OREGON STATE UNIVERSITY \\ UNIVERSITY OF OREGON \\ OSAKA UNIVERSITY \\ UNIVERSITY OF SOUTHERN CALIFORNIA
}

\author{
STANFORD UNIVERSITY \\ UNIVERSITY OF TOKYO \\ UNIVERSITY OF UTAH \\ WASHINGTON STATE UNIVERSITY \\ UNIVERSITY OF WASHINGTON \\ * * * * \\ AMERICAN MATHEMATICAL SOCIETY \\ CALIFORNIA RESEARCH CORPORATION \\ SPACE TECHNOLOGY LABORATORIES \\ NAVAL ORDNANCE TEST STATION
}

Mathematical papers intended for publication in the Pacific Journal of Mathematics should by typewritten (double spaced). The first paragraph or two must be capable of being used separately as a synopsis of the entire paper. It should not contain references to the bibliography. No separate author's resumé is required. Manuscripts may be sent to any one of the four editors. All other communications to the editors should be addressed to the managing editor, Richard Arens, at the University of California, Los Angeles, California 90024.

50 reprints per author of each article are furnished free of charge; additional copies may be obtained at cost in multiples of 50 .

The Pacific Journal of Mathematics is published quarterly, in March, June, September, and December. Effective with Volume 13 the price per volume (4 numbers) is $\$ 18.00$; single issues, $\$ 5.00$. Special price for current issues to individual faculty members of supporting institutions and to individual members of the American Mathematical Society: $\$ 8.00$ per volume; single issues $\$ 2.50$. Back numbers are available.

Subscriptions, orders for back numbers, and changes of address should be sent to Pacific Journal of Mathematics, 103 Highland Boulevard, Berkeley 8, California.

Printed at Kokusai Bunken Insatsusha (International Academic Printing Co., Ltd.), No. 6, 2-chome, Fujimi-cho, Chiyoda-ku, Tokyo, Japan.

PUBLISHED BY PACIFIC JOURNAL OF MATHEMATICS, A NON-PROFIT CORPORATION

The Supporting Institutions listed above contribute to the cost of publication of this Journal, but they are not owners or publishers and have no responsibility for its content or policies.

* Basil Gordon, Acting Managing Editor until February 1, 1966. 


\section{Pacific Journal of Mathematics}

\section{Vol. 15, No. $4 \quad$ December, 1965}

Robert James Blattner, Group extension representations and the structure space ........... 1101

Glen Eugene Bredon, On the continuous image of a singular chain complex .............. 1115

David Hilding Carlson, On real eigenvalues of complex matrices .................... 1119

Hsin Chu, Fixed points in a transformation group ............................. 1131

Howard Benton Curtis, Jr., The uniformizing function for certain simply connected Riemann

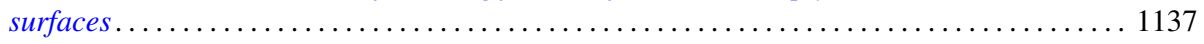

George Wesley Day, Free complete extensions of Boolean algebras................... 1145

Edward George Effros, The Borel space of von Neumann algebras on a separable Hilbert

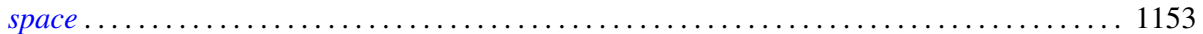

Michel Mendès France, $A$ set of nonnormal numbers ......................... 1165

Jack L. Goldberg, Polynomials orthogonal over a denumerable set ................ 1171

Frederick Paul Greenleaf, Norm decreasing homomorphisms of group algebras . . . . . . . . 1187

Fletcher Gross, The 2-length of a finite solvable group ........................ 1221

Kenneth Myron Hoffman and Arlan Bruce Ramsay, Algebras of bounded sequences ........ 1239

James Patrick Jans, Some aspects of torsion . . . . . . . . . . . . . . . . . . . . . . . 1249

Laura Ketchum Kodama, Boundary measures of analytic differentials and uniform

approximation on a Riemann surface ............................... 1261

Alan G. Konheim and Benjamin Weiss, Functions which operate on characteristic

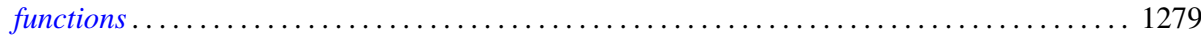

Ronald John Larsen, Almost invariant measures ............................ 1295

You-Feng Lin, Generalized character semigroups: The Schwarz decomposition ............ 1307

Justin Thomas Lloyd, Representations of lattice-ordered groups having a basis . . . . . . . . 1313

Thomas Graham McLaughlin, On relative coimmunity ....................... 1319

Mitsuru Nakai, $\Phi$-bounded harmonic functions and classification of Riemann surfaces ....... 1329

L. G. Novoa, On n-ordered sets and order completeness ..................... 1337

Fredos Papangelou, Some considerations on convergence in abelian lattice-groups . . . . . . . 1347

Frank Albert Raymond, Some remarks on the coefficients used in the theory of homology

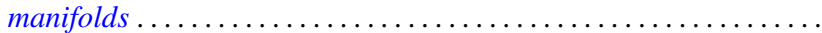

John R. Ringrose, On sub-algebras of a $C^{*}$-algebra .

Jack Max Robertson, Some topological properties of certain spaces of differentiable

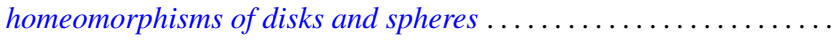

Zalman Rubinstein, Some results in the location of zeros of polynomials

Arthur Argyle Sagle, On simple algebras obtained from homogeneous general Lie triple systems. . . .

Hans Samelson, On small maps of manifolds ............................... 1401

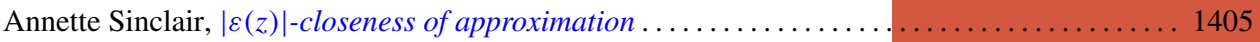

Edsel Ford Stiel, Isometric immersions of manifolds of nonnegative constant sectional curvature

Earl J. Taft, Invariant splitting in Jordan and alternative algebras ................. 1421

L. E. Ward, On a conjecture of R. J. Koch . . . . . . . . . . . . . . . . . . . . . . . . . . . 1429

Neil Marchand Wigley, Development of the mapping function at a corner . . . . . . . . . . 1435

Horace C. Wiser, Embedding a circle of trees in the plane ....................... 1463

Adil Mohamed Yaqub, Ring-logics and residue class rings . . . . . . . . . . . . . . . . 1465

John W. Lamperti and Patrick Colonel Suppes, Correction to: Chains of infinite order and their application to learning theory ........................................ 1471

Charles Vernon Coffman, Correction to: Non-linear differential equations on cones in Banach

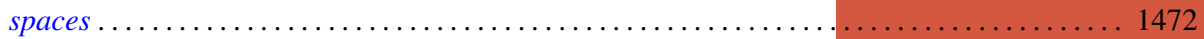

P. H. Doyle, III, Correction to: A sufficient condition that an $\operatorname{arc}$ in $S^{n}$ be cellular. . . . . . . . 1474

P. P. Saworotnow, Correction to: On continuity of multiplication in a complemented algebra 